\title{
Not Lost in Translation: Generalization of the Primary Systems Hypothesis to Japanese-specific Language Processes
}

\author{
Taiji Ueno $^{1}$, Satoru Saito ${ }^{1,2}$, Akie Saito ${ }^{3}$, Yuki Tanida ${ }^{2}$, \\ Karalyn Patterson ${ }^{4}$, and Matthew A. Lambon Ralph ${ }^{1}$
}

\begin{abstract}
The emergentist-connectionist approach assumes that language processing reflects interaction between primary neural systems (Primary Systems Hypothesis). This idea offers an overarching framework that generalizes to various kinds of (English) language and nonverbal cognitive activities. The current study advances this approach with respect to language in two new and important ways. The first is the provision of a neuroanatomically constrained implementation of the theory. The second is a test of its ability to generalize to a language other than English (in this case Japanese) and, in particular, to a feature of that language (pitch accent) for which there is no English equivalent. A corpus analysis revealed the presence and distribution of typical and atypical accent forms in Japanese vocabulary, forming a quasiregular domain. Consequently, according to the Primary Systems Hypothesis, there should
\end{abstract}

\section{INTRODUCTION}

The classic generative approach and its variants have equated language acquisition/use to the learning of and access to domain-specific forms of knowledge (i.e., rules) and whole-word representations (i.e., a mental lexicon; Pinker, 1991). An alternative view that has emerged through the development of connectionist models assumes domain-general, multiple, nonlinear probabilistic constraints on language acquisition/use. In these connectionist frameworks, language emerges from interaction between primary, general computational processing systems (the Primary Systems Hypothesis; Patterson \& Lambon Ralph, 1999; Seidenberg, 1997; Plaut, McClelland, Seidenberg, \& Patterson, 1996). For example, reading a written word aloud relies on an interaction across three primary systems: semantics, phonology, and vision/ orthography (Plaut et al., 1996). If this theoretical approach is a powerful one, then it should generalize (1) across verbal domains, (2) beyond the verbal domain, (3) and beyond English. Tests of the first two types of generalization have been conducted and yielded support for

${ }^{1}$ University of Manchester, ${ }^{2}$ Kyoto University, ${ }^{3}$ Doshisya University, Kyoto, Japan, ${ }^{4}$ University of Cambridge be a greater semantic impact on the processing of words with an atypical pitch accent. In turn, when word meaning is intrinsically less rich (e.g., abstract words), speakers should be prone to regularization errors of pitch accent. We explored these semantic-phonological interactions, first, in a neuroanatomically constrained, parallel-distributed processing model of spoken language processing. This model captured the accent typicality effect observed in nonword repetition in Japanese adults and children and exhibited the predicted semantic impact on repetition of words with atypical accent patterns. Second, also as predicted, in word repetition and immediate serial recall of spoken words, human participants exhibited reduced pitch-accent accuracy and/or slower RT for low imageability words with atypical accent patterns, and they generated accent errors reflecting the more typical accent patterns found in Japanese.

the primary systems of phonology and visual processing (Crisp \& Lambon Ralph, 2006; Behrmann, Nelson, \& Sekuler, 1998; Patterson \& Marcel, 1992). There is also growing convergent evidence that semantic information supports a wide range of verbal and nonverbal activities that are traditionally regarded as non- or presemantic (Woollams, Lambon Ralph, Plaut, \& Patterson, 2007; Patterson et al., 2006; Rogers, Lambon Ralph, Hodges, \& Patterson, 2004; Patterson, Lambon Ralph, Hodges, \& McClelland, 2001; Graham, Patterson, \& Hodges, 2000). These observations are of key theoretical relevance because, if correct, they place the semantic system at the heart of numerous verbal and nonverbal higher cognitive functions with implications for both cognitive and neuroscience theories (Holland \& Lambon Ralph, 2010; Patterson et al., 2006). The aim of the current study was to provide further evidence for the final part of the triumvirate-assessing generalization to non-English languages (Mirković, Seidenberg, \& Joanisse, 2011; Fushimi, Komori, Ikeda, Lambon Ralph, \& Patterson, 2009; Yang, McCandliss, Shu, \& Zevin, 2009) and, in particular, to assess how and when semantics supports processes in non-English languages.

Some key behavioral-computational details of the Primary Systems Hypothesis are founded on the more 
general principal of a division of labor between specific processes (e.g., translation of orthography to phonology for reading or acoustics to motor speech for repetition, which form a series of quasiregular domains; Seidenberg \& McClelland, 1989) and semantic representations. The division-of-labor hypothesis assumes that all word types are processed through an interaction between semantics and domain-specific systems. The involvement of semantics is present for all words (given that each has associated meaning), but the reliance on semantics for a specific verbal or nonverbal activity is graded and depends on the level of statistical ("surface") typicality in the inputoutput mapping of the domain (Woollams et al., 2007; Plaut et al., 1996). In tasks like reading words aloud, this semantic contribution is relatively redundant for frequent and typical patterns/mapping in each domain. This is because the modality-specific systems have acquired the surface-statistical structures of a given language (at both whole- and subword levels) during a lifetime of learning. As a result, they have been tailored toward the typical and frequent structures in that domain. In contrast, semantic input is especially useful, even indispensable, for atypical patterns/mappings because the modality-specific systems are particularly inefficient for these items. Furthermore, if the semantic contribution is minimized (see below), then the modality-specific systems cannot override the intrinsic tide of the learned statistical bias such that the resultant errors reflect more typical responses for the domain in question (i.e., regularization errors).

This semantic-phonology/-orthography interaction is a crucial element in a range of implemented computational models (Ueno, Saito, Rogers, \& Lambon Ralph, 2011; Welbourne, Woollams, Crisp, \& Lambon Ralph, 2011; Nozari, Kittredge, Dell, \& Schwartz, 2010; Woollams, Joanisse, \& Patterson, 2009; Dilkina, McClelland, \& Plaut, 2008; Woollams et al., 2007; Harm \& Seidenberg, 2004; Joanisse \& Seidenberg, 1999; Plaut et al., 1996; Seidenberg \& McClelland, 1989) and has been reinforced by studies of patients with semantic dementia, repetitive TMS in neurologically intact participants and by experimental manipulation of the intrinsic strength/richness of word meaning (imageability) in studies of past-tense verb generation (Butler, Patterson, \& Woollams, 2012; Holland \& Lambon Ralph, 2010), word reading (Woollams et al., 2007; Shibahara, Zorzi, Hill, Wydell, \& Butterworth, 2003; Strain, Patterson, \& Seidenberg, 1995), single-word repetition (Tyler, Voice, \& Moss, 2000), lexical decision (Evans, Lambon Ralph, \& Woollams, 2012; Tyler et al., 2000), verbal STM (Jefferies, Frankish, \& Lambon Ralph, 2006; Hulme et al., 1997), and across multiple tasks simultaneously (Patterson et al., 2006). We should note here that inefficiency/poor accuracy with atypical items cannot be explained simply in terms of these items being inherently more difficult to process. This is because other patient groups, with primary phonological impairment, exhibit a different pattern: no difference between known typical and atypical forms but poor processing of nonwords/ novel forms that are processed by unimpaired participants with reference to the domain's typical statistical structure (Rapcsak et al., 2009; Crisp \& Lambon Ralph, 2006; Patterson \& Marcel, 1992).

As noted above, two forms of supporting data have been sought for the primary systems account and, more specifically, for the importance of the semantic component: One is generalization across verbal tasks, and the other is verbal-nonverbal generalization (Woollams et al., 2007; Patterson et al., 2006; Rogers, Lambon Ralph, Hodges, et al., 2004; Patterson et al., 2001; Graham et al., 2000). The aim of the current study was to focus on the third element of the triumvirate-cross-language generalization, with particular reference to a language-specific (non-English) feature and the influence of semantic representation. Indeed, if this is a general theory about the interaction of surface representations with conceptual knowledge, then of course it should generalize beyond English and English-specific language phenomena (Lambon Ralph et al., 2011; Mirković et al., 2011; Yang et al., 2009). Some of these prior studies have focused primarily upon reading via a direct analogy with investigations of reading English words (Fushimi et al., 2009; Yang et al., 2009; Shibahara et al., 2003). The previous studies by Yang et al. (2009) are particularly relevant because the authors combined computational modeling and behavioral experiments to explore frequency and quasiregularity in Chinese reading. Indeed, although one might expect that Chinese character-based orthography requires different processing assumptions, Yang et al. were able to demonstrate that a standard PDP computational model (used in numerous explorations of English reading) was able to learn to read Chinese and generated predictions about the quasiregular statistics in Chinese reading that were closely matched by data from human participants. A similar dual-method approach was used to explore frequency and typicality in Japanese kanji reading (Ijuin, Fushimi, Patterson, \& Tatsumi, 1999). Other investigations have either explored a feature that is simple in English but considerably richer and more complex in other languages (e.g., inflectional morphology in Serbian; Mirković et al., 2011) or have made forays into language features that do not exist in English (e.g., grammatical gender of nouns in Spanish; Lambon Ralph et al., 2011). Despite the variety of languages and processing domains, all these studies have found results in support for a division of labor, namely that statistically atypical items are more difficult to process and, as a consequence, show the greatest benefit from semantic input and the greatest harm from its reduction in semantically impaired patients. As a further challenging test case, the current computational-plusempirical study broadened the cross-language exploration to investigate pitch accent, which is a specific feature of Japanese phonology that is not found in English. The following paragraphs will describe this aspect of Japanese phonology and the predictions generated with regard to its interaction with meaning. 
Spoken Japanese is considered to be a mora-timed rather than a syllable-timed language (Otake, Hatano, Cutler, \& Mehler, 1993). The mora is a subsyllabic spoken unit in Japanese. Morae include all of the following types of elements: a vocalic nucleus (V), a nucleus with onset (CV or CCV), a nasal consonant (N) in syllabic coda position, a geminate consonant, or a long vowel (Cutler \& Otake, 1994). Another aspect of Japanese phonology is pitch accent. Each multimoraic Japanese word has its own wordspecific pitch pattern (Akinaga \& Kindaichi, 2001). For example, the spoken form $/ n i-H O-n /$ (capital letters represent high pitch accent and small letters low pitch accent), meaning "Japan," has its pitch accent on the second mora, leading to a pitch accent pattern of low-high-low. The position of the pitch accent in a spoken word can sometimes uniquely determine its meaning (i.e., minimal accent pairs or homophones). For example, the spoken form /NI-bo-n/ means "two long, thin-shaped objects" (note that the moraic/phonemic forms are identical between / $\mathrm{ni}$-HO-n/ "Japan" and /NI-ho-n/ "two long, thin-shaped ..."). The fact that pitch accent is linked to word-specific information suggests the possibility that pitch accent processing, especially of lower-frequency words with less typical pitch patterns, may be strongly dependent on semantic information. Because Japanese pitch accent is not found in English and also because - compared with the orthography-phoneme/ mora translation underpinning reading - pitch accent is a more natural aspect of phonology, which children acquire without specific prompting, instruction, or teaching, Japanese pitch accent is a strong test case for the generalization of the primary systems hypothesis.

For a proper assessment, the first step in this approach is to establish the statistical structure of the processing domain in question (Lambon Ralph et al., 2011; Mirković et al., 2011). Pitch accent types do not occur equally frequently in Japanese (Kubozono, 2006; Sato, 1993). For example, there are four main types of pitch accent pattern for trimora nouns (Kindaichi, 2001), and Figure 1 shows the frequency distributions of the first three types, extracted from a contemporary psycholinguistic word fre-

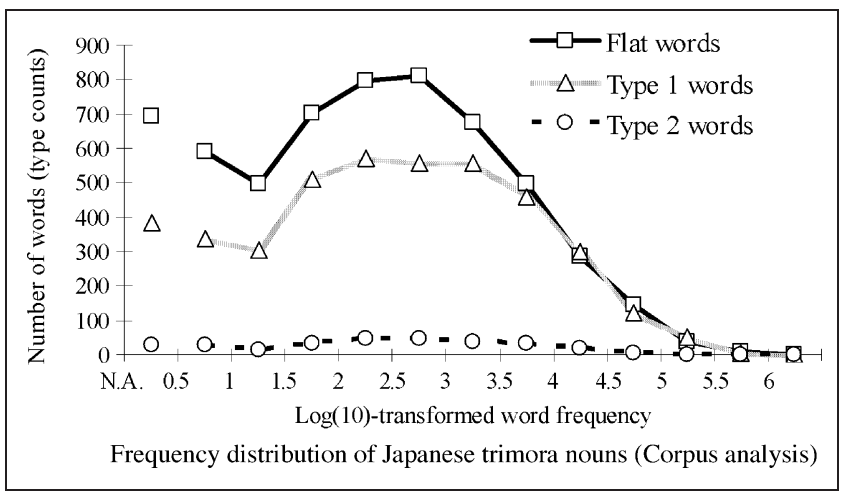

Figure 1. Accent typicality distributions from the corpus analysis. N.A. indicates that a word frequency value was not available from the database, probably reflecting the extremely low frequency of these items.
Table 1. The "On"/"Off" Status of One of the Units in the Input/Output Sound Layer

\begin{tabular}{lccc}
\hline & \multicolumn{3}{c}{ Accent Types } \\
\cline { 2 - 4 } & Flat-words & Type 1 Words & Type 2 Words \\
\hline First mora & Off & On & Off \\
Second mora & On & Off & On \\
Third mora & On & Off & Off \\
\hline
\end{tabular}

quency and accent type database (Amano \& Kondo, 1999, 2000). The most common is the flat accent pattern, which starts with a relatively low pitch mora followed by two relatively high pitch accented morae, for example, /to-KE-I/ (clock). This pattern is called "flat" because the pitch does not fall down within a word. In contrast, the following patterns have a within-word pitch fall. Also quite common are Type 1 accented words, whose spoken forms have their accent in the first mora, pronounced with a high-low-low pitch pattern (e.g., /KYO-O-to/ meaning Kyōto). Finally, the relatively uncommon pattern is Type 2 accented words, which mark the second mora with a high pitch accent creating a low-high-low accent pattern (e.g., /ni-HO-n/ meaning Japan). Type 3 words are very rare, so much so that they are not displayed in Figure 1. Thus, like many other aspects of English (Woollams et al., 2007; Patterson et al., 2006) and Japanese (Fushimi et al., 2009), Japanese pitch accent can be considered as another example of a quasiregular domain (Seidenberg, 1997; Plaut et al., 1996), in which the statistical structures of each language generate intrinsic, strong biases within each language process. Consistent with this notion, children's nonword repetition is more accurate for typically accented forms than atypical ones (Sakono, Ito, Fukuda, \& Fukuda, 2011; see Figure 3). One of the important assumptions of the connectionist approach is continuity between acquisition and processing (Seidenberg \& MacDonald, 1999). Accordingly, we would expect that an adult's phonological system is also biased toward the typical pitch accent pattern(s) and, as in other quasiregular domains, the contribution from semantics will be most important for words with an atypical pitch accent pattern. These predictions were tested through computational modeling and human behavioral experiments (Table 1).

\section{Study Components and Aims}

\section{Connectionist Modeling}

We have previously developed a neuroanatomically constrained model of normal and neurologically impaired (aphasic) spoken language processing (Ueno et al., 2011), which builds upon previous division-of-labor computational models (O’Reilly, 2006; Harm \& Seidenberg, 2004; Plaut et al., 1996; Seidenberg \& McClelland, 1989). The architecture of this neurocomputational model reflects a combination of the location of primary language-related brain 
regions and their white matter connectivity, thereby establishing both a dorsal pathway (important for sound-motor mapping) and a ventral pathway (central to the realization of sound-meaning-motor mappings; Rauschecker \& Scott, 2009; Saur et al., 2008; Hickok \& Poeppel, 2007; Parker et al., 2005). This model was trained to repeat, comprehend, and name/speak 1710 Japanese multisyllabic (three morae) words. Repetition, like the other language activities, was acquired via interaction between the two pathways. Like past models of this type, a division of labor emerges during training in this network, favoring a more prominent role for the dorsal over the ventral (semantically imbued) pathway for repetition over comprehension and naming. We used this same model in the current study to explore and generate testable predictions with regard to Japanese pitch accent processing. Following the primary systems hypothesis, we expected that repetition of words with an atypical pitch accent should be somewhat more reliant than typical patterns on activation from the ventral pathway. In addition, this model should be able to recapture both the developmental trajectory and adult performance on pitch accent processing (indexed by the accent typicality effect in nonword repetition) within a single framework.

\section{Human Experiments}

One of the advantages of implemented computational models is that they generate empirically testable hypotheses. Consequently, our core aim for the behavioral experiments was to explore the impact of semantics on pitch accent processing in three spoken language tasks (single-word repetition, repetition with accent correction, [Minematsu \& Hirose, 1995] and immediate serial recall; hereafter, repetition, correction, and ISR, respectively). Traditionally, repetition has been considered to occur essentially without reference to meaning (indeed, we are able to repeat meaningless nonwords) unless it has to overcome other phonologically similar competitors (Tyler et al., 2000). Past experiments have demonstrated that the impact of semantics on (English) language processing is more readily observed when the phonological activation is weaker/noisy/ambiguous (Jefferies, Crisp, \& Lambon Ralph, 2006; Tyler et al., 2000; Knott, Patterson, \& Hodges, 1997). In the current context, presentation of an atypical pitch accent pattern should lead to intrinsically weaker phonological activation because this pattern is much less frequent and, furthermore, experience with the more common typical pitch patterns will nudge the phonological system away from the atypical target pattern and toward the typical realization (Sakono et al., 2011). In addition, we utilized an experimental distortion of the pitch accent to stress the phonological system and thus make the contribution of semantics more obvious. Specifically, the stimulus was distorted by presenting it with an incongruent (incorrect) accent pattern (e.g., /ta-MA-go/ for egg is presented as /TA-ma-go/). In these experiments, we avoided all pitch accent minimal pairs. The pitch accent change, therefore, distorts the phonology of the target form whereas the moraic component still corresponds to the same target meaning. Congruently presented words (i.e., with the correct accent pattern) will be processed more efficiently by the phonological system because it has had substantial experience of these undistorted patterns before. In contrast, the incongruent pitch pattern is novel and will be processed less efficiently (for the incongruent presentation effect, see MasudaKatsuse, 2006). In this situation, we expected two things. First, the additional input from semantics should support the moraic phonological form and facilitate the ability of the phonological system to deal with these items although they are accompanied by a distorted pitch accent. Second, through an additional analysis of pitch accent transformations (see below), we also tested the hypothesis that semantic input would encourage an "autocorrection" of pitch accent. Indeed, Yuzawa (2002) demonstrated an accent congruency effect in younger ( 3 and 4 years old) children's phonemic/moraic recall accuracy of spoken word lists, which was not the case of the older children (5 and 6 years old) whose phonological and semantic systems are better developed, and at this stage, the older children were found to "autocorrect" the incorrectly presented pitch accents. Finally, we also included standard serial order recall in which multiple words are presented before the participants repeat the word list. Again, past studies have shown that repetition of multiple words increases the influence of semantic variables (Jefferies, Frankish, et al., 2006).

\section{METHODS}

\section{Simulation}

Figure 2A summarizes the architecture of the neuroanatomically constrained PDP model (Ueno et al., 2011). The model was implemented and trained with the LENS software (Rohde, 1999) and used important implementation techniques developed for prior computational models, encapsulated by the primary systems hypothesis (Welbourne et al., 2011; Nozari et al., 2010; Dilkina et al., 2008; Woollams et al., 2007; Botvinick \& Plaut, 2006; Rogers, Lambon Ralph, Garrard, et al., 2004; Plaut \& Kello, 1999; Dell, Schwartz, Martin, Saffran, \& Gagnon, 1997; Plaut et al., 1996; McClelland, St John, \& Taraban, 1989; Seidenberg \& McClelland, 1989). Readers are referred to Ueno et al. (2011) for full implementation details. Key features and novel implementation steps are summarized below. All other parameters and implementation details were the same as reported by Ueno et al. (2011).

We utilized the same computational framework to explore a range of additional language features/behaviors within the same general theoretical approach. While inheriting important features of other "triangle models," this architecture also incorporates a range of neuroanatomical 


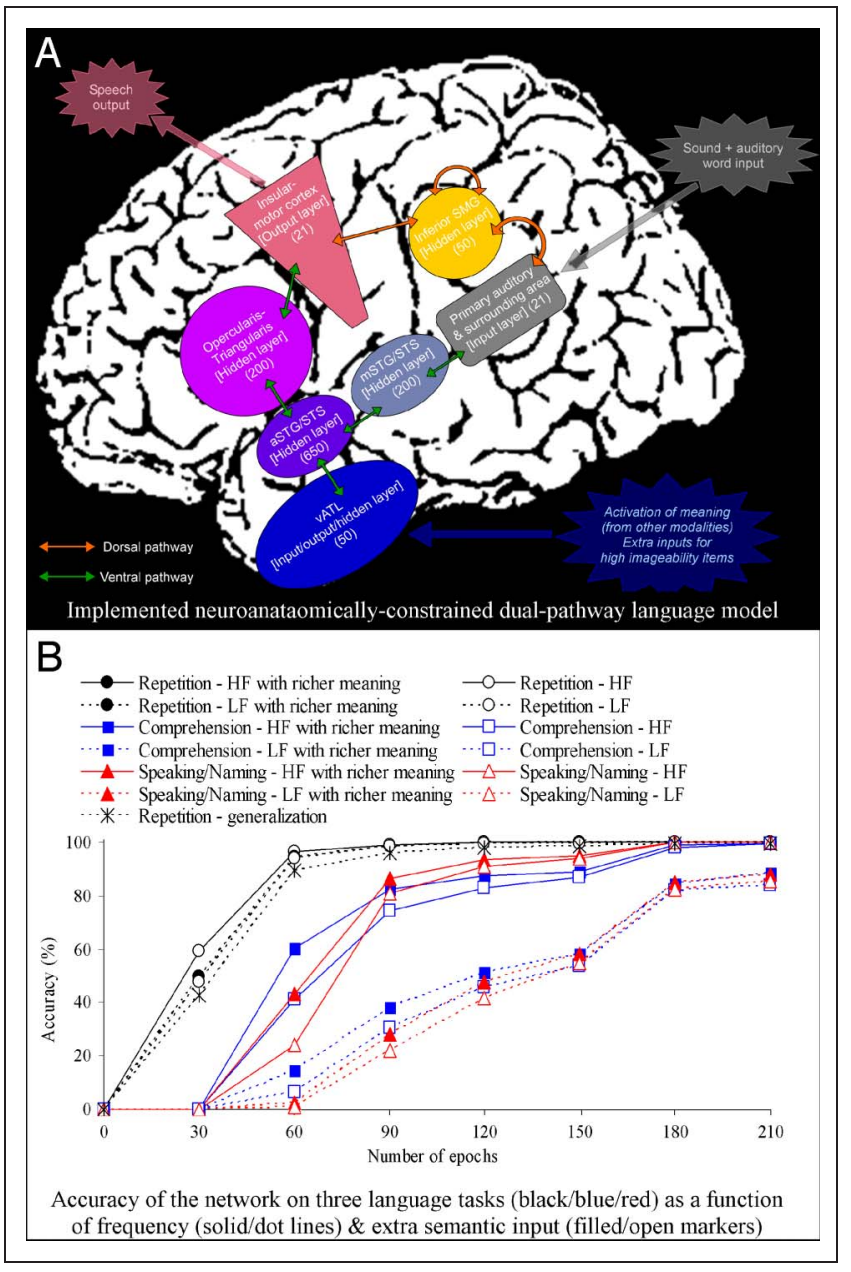

Figure 2. Implemented neuroanatomically constrained dual-pathway language model (top, A) and its accuracy during training (bottom, B). Abbreviations: $\mathrm{HF} / \mathrm{LF}=$ high/low frequency. See main text and Methods for further details on training and testing the model.

constraints (including dorsal and ventral language pathways) that allow it to simulate both neuropsychological and neuroimaging findings in addition to those reported in the current study (Ueno et al., 2011). The model was trained to complete three different language activities: (a) word repetition (taking the time-varying, mora-by-mora, phonological input and, once finished, generating the same pattern as a time-varying output); (b) word comprehension (taking the time-varying phonological input and converting it to a stable, time-invariant semantic representation as soon as possible); (c) speech production (taking the learned time-invariant semantic representation and converting it to a time-varying phonological output). The phonological input and output representations were identical and the 21-bit distributed representations coded both the mora and pitch accent elements of each word. The training set comprised the same 1710 items (each three morae in length) as Ueno et al. (2011), selected from the NTT Japanese psycholinguistic database (Amano \& Kondo, 1999). These items crossed two word frequency bands (high and low) with three pitch accent types (1000 flat items, 660 Type 1 items, and 50 Type 2 atypical items) to mimic the statistical structure of Japanese phonology (see Figure 1). The remaining 3511 untrained trimora items in the NTT corpus were used for testing generalization (the asterisk marker line in Figure 2B).

To investigate the effect of semantics on pitch accent processing, we divided the training items into two sets (matched for frequency and pitch accent types). One half received extra input to the semantic layer during training. Specifically, following the dual-coding theory (Paivio \& Madigan, 1968), we assumed that words with richer meaning (e.g., highly imageable words) are encoded both in the verbal and visual domains. Thus, in this spoken language model, the semantic layer received extra input (adding 0.5 to the net input of the to-be-on units) to approximate the contribution from the visual system, such that the output was moved toward the correct semantic pattern (Plaut et al., 1996). The key issue in this study was whether or not the network utilized this extra semantic boost (applied to the semantic layer within the ventral pathway) for generating the correct pitch accent pattern in the speech output layer during repetition (which is primarily computed in the dorsal pathway; see Ueno et al., 2011). The data shown in Figure $4 \mathrm{~A}$ and $\mathrm{B}$ were computed from the results on 150 experimental items from ten replications of the model (25 items in each the six conditions, formed by crossing three accent types and two with/without extra semantic input, controlled for word frequency).

\section{Human Experiments}

\section{Participants}

One hundred fifty-two Kyoto university students were tested individually and were paid for their participation (see Table 2 for the mean ages). All the participants were

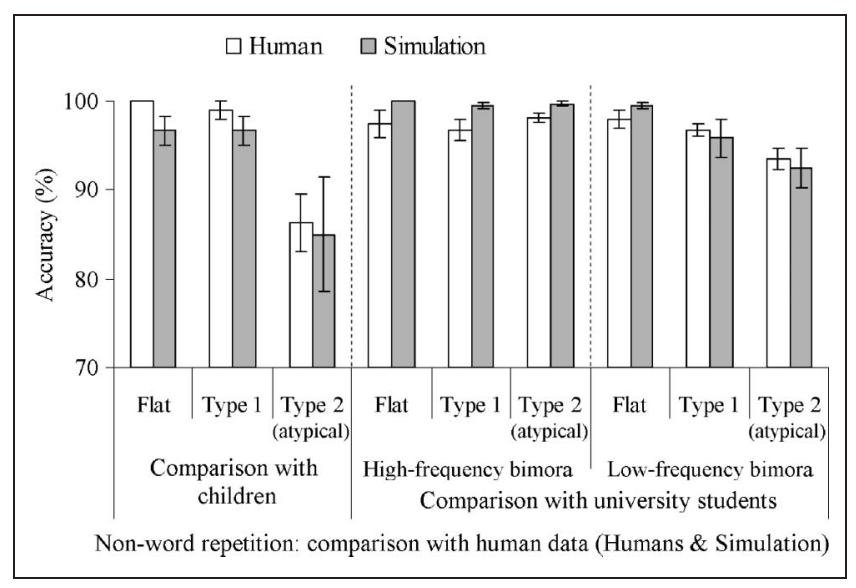

Figure 3. Accent typicality effect on nonword repetition in human children versus simulation (first panel on the left) and in adults versus simulation (middle and right panels). The human developmental data (left panel) are from Sakono et al. (2011). 
native Japanese speakers with no reported history of speech or hearing difficulties.

\section{Nonword Repetition (Figure 3)}

Each of 36 high/low bimora frequency (Tamaoka \& Makioka, 2004) Japanese nonwords was presented three times (once per block). In each block, one of the three pitch accent patterns was assigned. The participants were required to repeat the presented nonword with the same accent pattern without time pressure. A real word was inserted occasionally (11.1\% chance) as a dummy item to encourage natural repetition and the avoidance of any abnormal strategy. Other procedures are summarized in the right column of Table 2 .

\section{Word Repetition/Correction/ISR (Figure 5)}

Table 2 shows the characteristics of the materials. In each cell of the six conditions ( 3 Accent Types $\times 2$ Levels of Imageability), 40-44 items were selected from the NTT psycholinguistic database (Amano \& Kondo, 1999, 2000). The materials are provided in the SI Appendix. Pitch accent varies somewhat across different regions in Japan. For more common words, it is relatively consistent and fits with the accent pattern coded in the NTT psycholinguistic database (sampled from the standard Japanese dialect in Tokyo district; Amano \& Kondo, 1999). In addition, we screened the materials using a strict two-step procedure. First, words were only selected if they have $100 \%$ accent position validity, which reflects the level of interrater agreement on the accent position of a word (Amano \& Kondo, 1999). Homophony was checked by screening this corpus for any pairs of words with the same phoneme/mora sequence and any homophonous items were removed. (b) Second, words were only selected if $100 \%$ of the participants in the NTT database rated the item as known (rather than unfamiliar or unknown on a 3-point Likert scale; Amano \& Kondo, 1999). As an additional check, 120 selected experimental items were screened and were found to have no variation in pitch accent in participants from different Japanese regions (Kinki/Tokai/Chugoku/Kyusyu/Kanto/Shikoku areas: mean consistency $=98.25 \%$; $S D=2.86 \%$ ).

In repetition/correction, the numbers of items with a given onset type (i.e., the initial consonant/vowel) were matched across the Accent Type $\times$ Imageability conditions, a variable known to affect RT (Kawamoto, Kello, Jones, \& Bame, 1998).

\section{Accent Congruency}

Half of the items were presented with an incongruent (incorrect) pitch accent pattern (e.g., /ta-MA-go/, which is the correct pattern for egg, is presented as /TA-ma-go/).
This accent change does not alter the phonemic form of the item, and because we avoided homophones, the meaning is also unchanged. The congruent/incongruent trials were randomly distributed in repetition/correction. In ISR, all five words within a single list were selected from the same imageability range (high or low) and had the same accent presentation (congruent or incongruent). Allocation of congruent/incongruent pattern to each item was fully counterbalanced across participants. Thus, across all participants, the accent congruency factor was manipulated within the same set of materials.

\section{Procedure}

The bottom line of Table 2 shows the flow of a trial. The stimulus presentation was controlled by the HSP (Hot Soup Processor) experimental program (hsp.tv/). The participants were required to repeat the phoneme (mora) sequence as quickly as possible (a) with the presented accent pattern in repetition (be it congruent or incongruent) and (b) with the congruent accent pattern in correction. (c) In ISR, a quick response was not required. At 1200 msec after the fifth stimulus, a beep sounded to indicate that the participant should recall the five words in the presented order. When the participants could not remember a word in a given serial position, they were asked to tap the desk with a finger to mark the missing word. A rest was interpolated halfway through the experiment. Each word, with either the congruent (correct) or incongruent pitch pattern, was digitally recorded by a male speaker. In repetition/correction, the duration of the spoken form of all the trimora words was fixed at $600 \mathrm{msec}$ by sound editing software (Audacity, audacity.sourceforge. net/). RT was measured from the onset of each stimulus.

\section{Instructions}

In all three tasks, a spoken word was presented either with the correct (congruent) pitch accent or with the incorrect (incongruent) pitch accent. In repetition, the participants were required to repeat the phonemic/moraic sequences with the presented accent pattern (correct or incorrect). In the correction task, participants were asked to repeat the word with the correct pitch accent (thus having to correct items which had been presented with the incorrect pitch pattern). (c) In ISR, five spoken words were presented and the participants were required to recall the items in the presented order. No specific instructions were provided as to the desired output accent pattern so that the results were directly comparable to a previous developmental study (Yuzawa, 2002), where older children spontaneously corrected the incongruent accent pattern. We expected the rate of autocorrection in the ISR task to be more common for higher than lower imageability words. 
Table 2. Demographics of the Participants, Item Characteristics, and a Flow of a Trial in Human Experiments

\begin{tabular}{|c|c|c|c|c|c|}
\hline Task & Repetition (Figure 5) & $\begin{array}{l}\text { Accent Correction } \\
\text { (Figure 5) }\end{array}$ & ISR (Figure 5) & ISR (Pilot Study) & $\begin{array}{c}\text { Nonword Repetition } \\
\text { (Figure 3, Middle and Right) }\end{array}$ \\
\hline No. of participants & 20 & 20 & 64 & 36 & 12 \\
\hline Mean age $(S D)$ & $23.15(3.45)$ & $22.35(3.54)$ & $20.98(2.85)$ & $22.97(3.54)$ & $20.33(1.87)$ \\
\hline Factorial design & \multicolumn{3}{|c|}{3 Accent Types $\times 2$ Word Imageability } & 2 Word Frequency & 3 Accent $\times 2$ Bimora Frequency \\
\hline No. of items per a condition ${ }^{a}$ & \multicolumn{2}{|l|}{44} & 40 & 100 & 36 \\
\hline High imageability items, mean $(S E)$ & \multicolumn{2}{|c|}{$5.08(0.05)$} & $5.66(0.05)$ & - & - \\
\hline Low imageability items, mean (SE) & \multicolumn{2}{|c|}{$3.51(0.04)$} & $3.21(0.03)$ & - & - \\
\hline Log10-transformed word frequency $(S E)^{\mathrm{b}}$ & \multicolumn{2}{|c|}{$2.06(0.03)$} & $2.43(0.08)^{\mathrm{c}}$ & $1.66(0.03)-3.94(0.05)^{\mathrm{d}}$ & $-{ }^{\mathrm{e}}$ \\
\hline Accent validity & \multicolumn{2}{|l|}{$100 \%$} & $100 \%$ & $100 \%$ & - \\
\hline Familiarity of the items (3-point Likert) & \multicolumn{2}{|c|}{ Maximum } & Maximum & Maximum & \multirow[b]{2}{*}{$100 \%$} \\
\hline Phoneme (mora) dictation accuracy (SE) & \multicolumn{2}{|c|}{$90 \%(2 \%)^{f}$} & $99 \%(0.1 \%)$ & $89 \%(1 \%)$ & \\
\hline Mora length & \multicolumn{4}{|c|}{3} & \\
\hline No. of items in a trial & \multicolumn{2}{|l|}{1} & \multicolumn{2}{|l|}{5} & 1 \\
\hline No. of practice trials & \multicolumn{2}{|l|}{6 items } & \multicolumn{2}{|c|}{4 list (20 items) } & 9 items \\
\hline Flow of a trial & \multicolumn{2}{|c|}{$\begin{array}{l}\text { A beep }+ \text { silence }(500 \mathrm{msec})+ \\
\text { single word }(600 \mathrm{msec} \text { duration })+ \\
\text { repeat as quickly as possible }\end{array}$} & \multicolumn{2}{|c|}{$\begin{array}{l}\text { A beep }+ \text { silence }(1200 \mathrm{msec})+ \\
5 \text { words }(1200 \mathrm{msec} \text { SOA })+ \\
\text { serial recall }(10 \mathrm{sec})\end{array}$} & $\begin{array}{l}\text { Press space bar }+ \text { silence }(500 \mathrm{msec})+ \\
\text { single nonword }+ \text { repeat without } \\
\text { time pressure }\end{array}$ \\
\hline
\end{tabular}

${ }^{a}$ The identical set of stimuli was used in repetition and in correction.

${ }^{\mathrm{b}}$ The total sample size of the NTT word frequency assessment was approximately 240 million words.

${ }^{\mathrm{c}}$ Frequency was manipulated, yet very weakly (Cohen's $d=0.9$ for the manipulation size) due to the severe limitation in the number of items available. See Note d, below.

${ }^{\mathrm{d}}$ Frequency was strongly manipulated in this pilot study (Cohen's $d=6.0$ ), and its interaction with accent congruency was formally investigated (see Figure S3).

${ }^{e}$ Bimora frequency (Tamaoka \& Makioka, 2004) was manipulated (high-frequency bimora: mean $=149,009, S D=28,066$; low-frequency bimora: mean $=1764, S D=471$ ).

${ }^{\mathrm{f}}$ After this dictation check, any item with less than $50 \%$ accurate was re-recorded. 


\section{RESULTS}

\section{Connectionist Modeling}

\section{Learning}

Figure 2B shows the developmental trajectory of the network on three tasks. In each case, the network achieved equivalent performance to humans (for the comparison with human normative data, see Ueno et al., 2011).

\section{Nonword Repetition of Children and Adults}

As shown in Figure 2B, the model acquired the statistical structure that exists between speech sounds and articulatory gestures, such that it was able to repeat not only all of the words from the training set but also other untrained items (generalization; see asterisk marker line in Figure 2B). Figure 3 compares the nonword repetition results from human behavioral experiments with those from the simulation. During training (150th epoch), the model captured the accent typicality effect, $F(2,18)=$ $4.23, p=.03$, that was observed in the developmental literature (Sakono et al., 2011; see Figure 3, left). In addition, the adult model (i.e., after the training) also captured the small, yet significant accent typicality effect for the low frequency bimora nonword condition, $F(2,18)=4.96$, $p=.02$, that was also observed in the human experiment, $F(2,22)=7.87, p<.01$ (see Figure 3, middle and right).

\section{Semantic $\times$ Pitch Accent Interaction}

The network's nonword repetition performance (Figure 3) indicated, as expected, an intrinsic bias toward the typical pitch accent pattern. We predicted, therefore, that repetition of words with an atypical pitch accent pattern would rely more on additional semantic input than typically accented words. Figure 4A shows the network's degree of production error (the difference between the target and output values) for the different word types. Following the assumptions made in prior simulations (Woollams et al., 2009; Harm \& Seidenberg, 2004; Plaut \& Kello, 1999; Seidenberg \& McClelland, 1989), this measure was taken to reflect the network's confidence (smaller is more confident) and an analogue of human RTs. The network exhibited smaller production error for words with typical pitch accent patterns while additional input from the semantic layer in the ventral pathway (an analogue of words with concrete/richer meaning; Paivio \& Madigan, 1968) made no difference for these words. In contrast, this semantic manipulation significantly reduced the production error for words with an atypical pitch accent pattern [for production error with/without extra semantic input interaction, $F(2,18)=13.18, p<.01$; for the extra input effect in the Type 2 condition, $F(1,9)=14.70, p<$ $.01]$. A control simulation revealed that the difference between these two conditions (with/without extra inputs for Type 2 accent items) disappeared (and even went

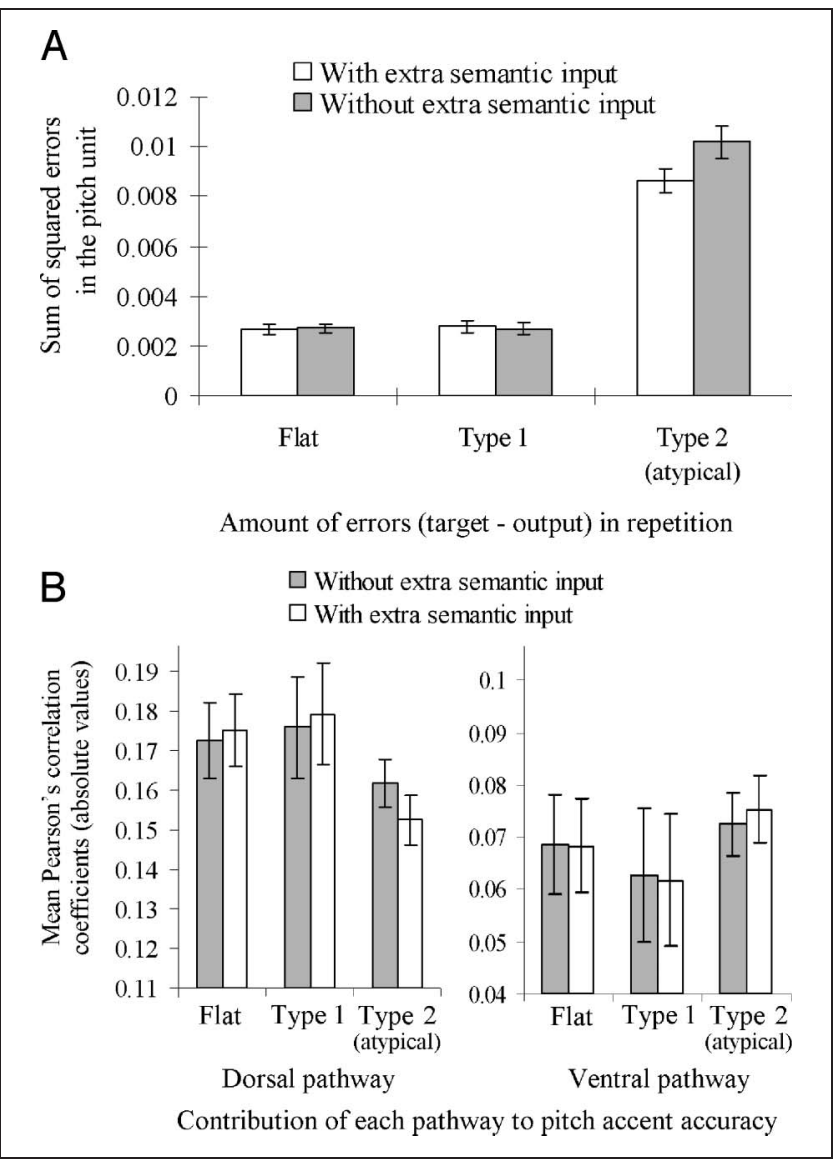

Figure 4. Simulation findings. (A) The effect of extra semantic input (a computational analogue of concrete $>$ abstract concepts) on repetition of words with different accent types. (B) Contribution of each network pathway to pitch accent accuracy as a function of accent typicality (see main text for explanation).

somewhat in the opposite direction, $p=.25$ ) if we did not provide any extra semantic input (i.e., a set of control simulations with the same random initial seeds but without any manipulation of the extra semantic input). This suggests that our manipulation (extra semantic input) had a causal effect on the production error for Type 2 words.

\section{Diagnostic Analysis of the Network's Internal Knowledge}

Figure $4 \mathrm{~B}$ visualizes the differential contributions to the pitch accent output from the dorsal and the ventral (mainly semantic) pathways, as a function of accent typicality and with/without extra semantic input. The $y$ axis represents the absolute value of the Pearson's correlation between the weight vector coming into the pitch accent unit and the activation vector in the previous internal layers of the dorsal and the ventral pathways. A higher correlation between these two vectors indicates a higher absolute value of the net input, denoting that the pathway makes a strong contribution to the output value on the pitch unit (see Botvinick \& Plaut, 2006, for further 
details on this analysis method). A three-way interaction $(3$ Accent Types $\times 2$ Pathways $\times 2$ With/Without Extra Semantic Inputs) was significant, $F(2,18)=14.49, p<$ .01 . The higher values from the dorsal pathway (left half) than from the ventral pathway [right half; note the $y$-axis scale is different; $F(1,9)=27.40, p<.01]$ support the key role of the dorsal pathway in repetition and pitch accent statistics (Ueno et al., 2011; Fridriksson et al., 2010). This division of labor, however, shifted somewhat toward the ventral pathway for the atypical pitch accent pattern (i.e., reduced contribution in the dorsal pathway and increased in the ventral pathway for Type 2 patterns). This shift was particularly clear for the items with extra semantic input (white bars), $F(2,18)=4.98, p=.02$. With extra semantic input, there was a significant effect of accent type on this correlation measure in the ventral pathway (Figure 4B, right), $F(2,18)=4.56, p=.03$. In summary, these results suggest that the internal structure of the ventral (semantic) pathway was tailored such that the strong input from semantics helped computation of the atypical pitch accent pattern.

\section{Human Experiments}

Figure 5 shows the pitch accent accuracy (for items which were produced with the correct phonemes/morae) and RTs for correct responses in the three language tasks. The ISR results were identical whether item accuracy was coded irrespective of recall position or in terms of a stricter correct-in-serial position criterion (the latter data are provided in Figure S2).

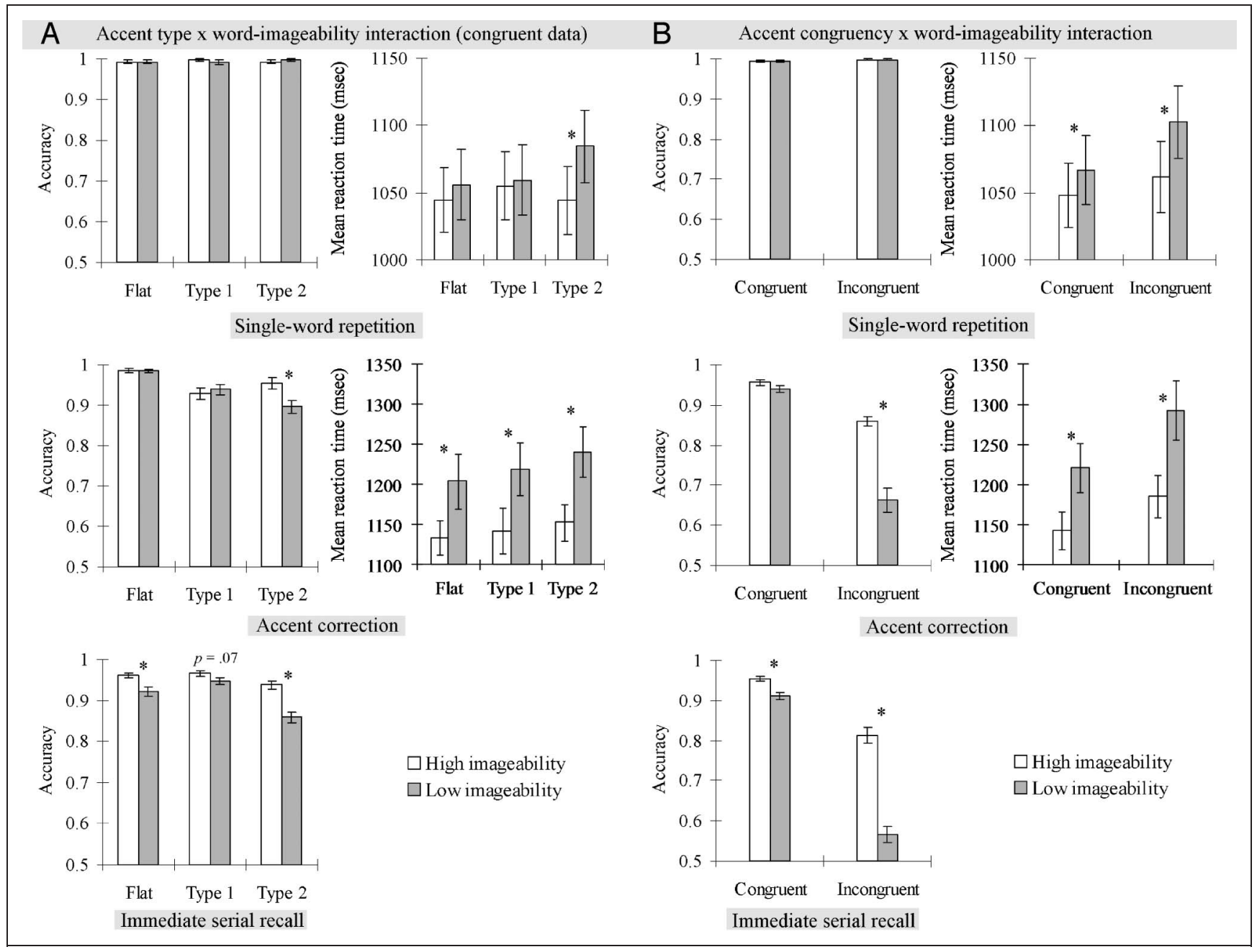

Figure 5. The effects of accent type, imageability, and presentation form on three tasks: single-word repetition (top third), accent correction (middle third), and ISR (bottom third). Accuracy and RT are displayed for repetition and correction; ISR performance is only measured in terms of accuracy. Given the large volume of data, the results are shown in two forms: (A, left-half) The relationship between accent typicality and imageability just for the congruently presented trials. As expected, across all three tasks, performance was worst (slower and/or less accurate) for words with an atypical Type 2 accent pattern, and this effect was exacerbated for words with intrinsically weak meaning (low imageability). (B, right-half) Summarizes the effect of accent congruency for high or low imageability. Again, as expected, performance was worse in the incongruent condition, and this difference was augmented for low imageability items. * denotes a significant imageability effect. 


\section{Accent Typicality $\times$ Semantics Interaction}

The left half of Figure 5 (A) highlights the interaction between pitch accent typicality and imageability (an index of the intrinsic strength of word meaning) obtained in the congruently presented condition of the three tasks (i.e., trials in which the item was presented with its correct pitch accent). Given that repetition is a relatively easy language task (99\% accuracy), effects of the experimental variables were shown purely in RTs [Typicality $\times$ Imageability interaction: $F(2,38)=4.62, p=.02]$. Subsidiary analysis showed a significant imageability effect for atypical pitch accent items, $F(1,19)=13.15, p<.01$. On the more demanding correction and ISR tasks, significant Imageability $\times$ Accent interactions were obtained on accuracy [correction: $F(2,38)=5.81, p<.01$; ISR: $F(2,126)=6.72, p<$ $.01]$. Subsidiary analysis revealed that the imageability effect was the strongest for words with atypical (Type 2) pitch accent patterns [correction: $F(1,19)=14.74, p<$ .01 ; ISR: $F(1,63)=28.00, p<.01]$. The imageability effect on correction RTs was pervasive across all the accent types, $F(1,19)=62.45, p<.01$.

\section{Accent Congruency $\times$ Semantics Interaction}

The right half of Figure 5 (B) highlights the interaction between pitch accent congruency and imageability. The repetition and correction $\mathrm{R}$ Ts exhibited a significant Accent Typicality $\times$ Imageability interaction [repetition: $F(1,19)=5.40, p=.03$; correction: $F(1,19)=5.48$, $p=.03]$. The imageability effect was larger for the incongruently presented items in both repetition (40 msec) and correction (105 msec) than for the congruently presented items (18 and $78 \mathrm{msec}$, respectively). In a similar vein, accuracies showed a significant Accent Typicality $\times$ Imageability interaction in the correction task, $F(1,19)=$ $34.11, p<.01$, and in ISR, $F(1,63)=149.58, p<.01$. The imageability effect on accuracy was larger for the incongruently presented words in correction (18\%) and in ISR (24\%) than for the congruently presented words (1\%, ns, and 4\%, respectively).

\section{Accent Typicality Shift in Errors}

As summarized in Figure 5, in the two more demanding tasks (correction and ISR), participants generated a small number of pitch accent errors even when the stimuli were presented with a correct pitch accent (congruent condition). This allowed us to explore whether the distribution of pitch accent errors reflects the accent typicality of Japanese phonology and how this interacts with imageability (intrinsic semantic strength). The left and middle panels (congruent condition) of Figure 6 summarize the error rates, splitting them into two types: (a) more atypical responses - where an error reflects a more atypical pitch pattern than the presented item (flat $\rightarrow$ Type 1 or Type 2 ; or Type $1 \rightarrow$ Type 2 ) versus (b) more typical responses, which follow the opposite transformation and are in line with the statistical bias inherent in the language (Type 1 or Type $2 \rightarrow$ flat; or Type $2 \rightarrow$ Type 1 ). In both tasks, the direction of the pitch accent errors interacted with imageability [correction: $F(1,19)=10.01, p<.01$; ISR: $F(1,63)=$ $6.46, p=.01]$. Subsidiary analysis revealed a higher rate of typically transformed errors (a form of regularization) than of atypically transformed errors for low imageability words [correction: $F(1,19)=17.64, p<.01$; ISR: $F(1$, $63)=20.14, p<.01]$. In contrast, this bias was not significant for high imageability words in correction $(F<1)$ and was attenuated in ISR, $F(1,63)=5.09, p=.02$. Finally, the same analysis was conducted on the data in the incongruent condition of the correction task (Figure 6, right), which required the participants to correct the pitch accent. The same pattern emerged with the rate of typically transformed errors being more frequent than atypically transformed errors for low imageability words, $F(1$,

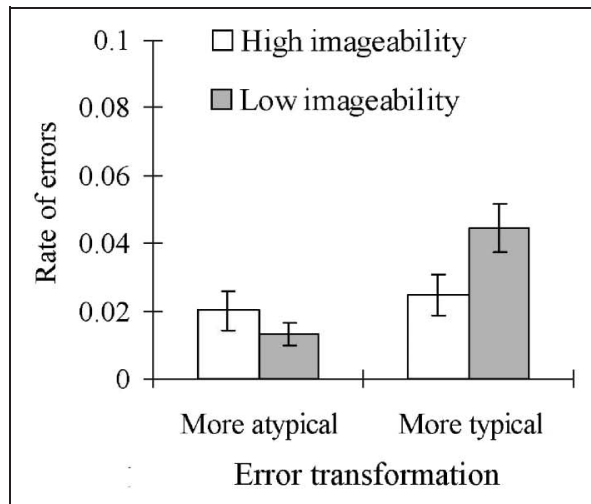

Accent correction (congruent condition)

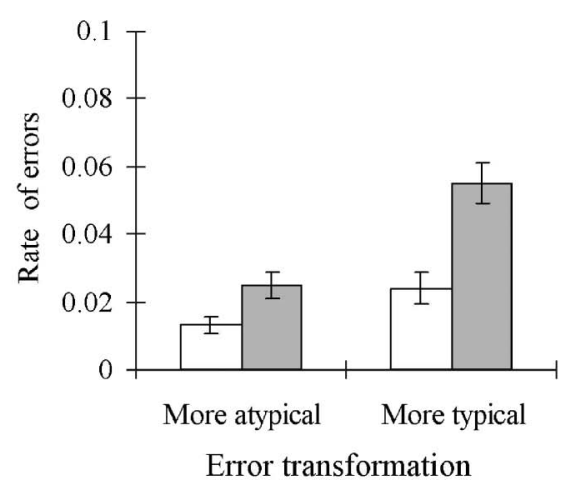

Immediate serial recall (congruent condition)

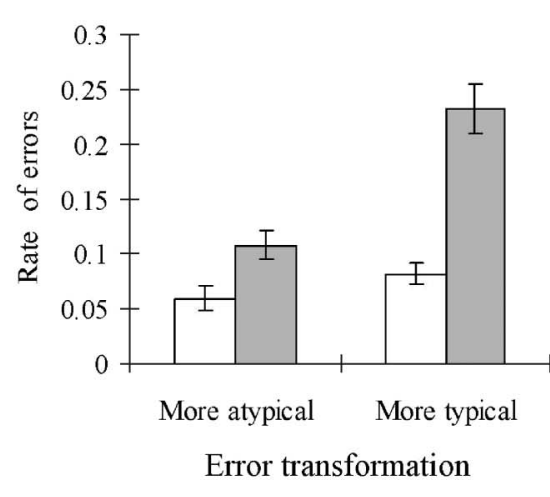

Accent correction (incongruent condition)

Figure 6. The rate of different pitch accent error types as a function of imageability. Each graph contrasts errors that led to either a less typical or more typical pitch accent pattern. In both ISR and accent correction, a strong pitch accent bias toward a more typical realization was revealed, especially for words with intrinsically weak meaning (low imageability). 
19) $=34.85, p<.01$, whereas this difference disappeared for high imageability items, $F(1,19)=2.05, p=.16$.

\section{DISCUSSION}

Using a multimethod approach (computational modeling and human behavioral experiments), this investigation found evidence for cross-language generalization of the Primary Systems Hypothesis (Crisp \& Lambon Ralph, 2006; Harm \& Seidenberg, 2004; Patterson \& Lambon Ralph, 1999; Plaut et al., 1996; Seidenberg \& McClelland, 1989). This emergentist-connectionist approach assumes that language activities are underpinned by interaction between primary neural systems for semantics, phonology, vision, and so on. To date, the applicability of this theory has been demonstrated across different (English) language activities (e.g., reading, spelling, past tense verb generation, etc.) and shown to generalize across verbal and nonverbal domains (Woollams et al., 2007; Patterson et al., 2001, 2006; Rogers, Lambon Ralph, Hodges, et al., 2004; Graham et al., 2000). The data in this study provide further support for the third and final part of this triumvirate by demonstrating that this computationally implemented theoretical framework generalizes to a feature of the Japanese language that has no English analogue.

Pitch accent is a core component of Japanese phonology, which children acquire without specific prompting, instruction, or teaching. It has a statistical structure that forms a quasiregular domain, an aspect which can be found repeatedly across languages and across different elements of any one language (Seidenberg, 1997; Plaut et al., 1996). Previous studies have demonstrated that the contribution of semantics is especially critical for processing words that contain elements that run counter to the language-wide statistical tendency (Seidenberg \& MacDonald, 1999; Seidenberg, 1997) and so we predicted that, in Japanese, processing of words with an atypical pitch accent would reveal a greater contribution from semantics.

Computational models of various English-language activities have encapsulated the ideas of the Primary Systems Hypothesis and demonstrated the differential importance of semantics for atypical words. We extended these models, for the first time, to Japanese via a neuroanatomically constrained computational model (Ueno et al., 2011). Such implementations allow the computational details of any cognitive theory to be made explicit and evaluated. Indeed, this model shows that English implementations of the Primary Systems Hypothesis can be successfully extended to Japanese and can capture non-English features such as pitch accent. In the context of the current study, we demonstrated that the model was able to learn three core language activities, including repeating words with the correct pitch accent, and to generalize this statistical knowledge to novel items (nonword repetition). Detailed investigation of the model indicated that (a) the dorsal language pathway is especially impor- tant for word and nonword repetition but that (b) the graded division of labor (Welbourne et al., 2011; Woollams et al., 2007; Harm \& Seidenberg, 2004; Plaut et al., 1996) is partially shifted toward the ventral (semantically related) pathway when repeating words with atypical pitch accents.

Having implemented the Primary Systems Hypothesis within a neurocomputational model, we explored the resultant hypothesis that the semantic contribution for words with atypical pitch accents should be especially important in human performance. This hypothesis was investigated and supported three times via different spoken language tasks (repetition, correction, and immediate serial recall). In all tasks, we manipulated accent typicality and congruency (i.e., manipulation of the surface level characteristics) to maximize the detection power for the contribution of semantics (Lambon Ralph et al., 2011; Patterson et al., 2006). The consistent finding from these three different tasks was that, as expected, the influence of a word's intrinsic semantic richness (imageability) was especially apparent when repeating/recalling words with an atypical pitch accent. This effect was present in simple, single word repetition but was augmented when the production task was more demanding (e.g., repetition of multiple words). A second crucial prediction from the Primary Systems Hypothesis and associated model was also supported. Specifically, when participants made a pitch accent production error, they were much more likely to produce a more typical pitch accent (a form of regularization error), consistent with the influence of the underlying phonological statistics of the language. These results reinforce two core tenets of the Primary Systems Hypothesis, that is, language performance reflects the intrinsic characteristics/statistics of each primary system and their interactivity. Demonstration of these effects even in single-word repetition is a particularly striking example of the generalization to Japanese. This is because the ability to repeat is a natural, automatic language activity and one that is acquired very early by children without direct instruction, implying that the Primary Systems Hypothesis provides an important theoretical framework for all types of language activity.

The implemented computational models of the Primary Systems Hypothesis not only demonstrate its computational adequacy but also help to explain how language activities can emerge from the interaction between the primary systems. The models indicate that each activity/ task is underpinned by a direct transformation from input to output (e.g., heard speech $\rightarrow$ speech output for repetition; visual processing $\rightarrow$ speech output for reading) combined with an interaction with meaning (Lambon Ralph et al., 2011; Welbourne et al., 2011; Woollams et al., 2007; Patterson et al., 2001, 2006; Harm \& Seidenberg, 2004; Rogers, Lambon Ralph, Hodges, et al., 2004; Graham et al., 2000; Patterson \& Lambon Ralph, 1999; Plaut et al., 1996). The exact balance across these two elements (the division of labor) reflects the difficulty of the direct 
mapping and the underlying statistics of the domain such that semantic input is more important and apparent in empirical studies when the required input $\rightarrow$ output transformation is opaque. In quasiregular domains, the direct mapping allows the computational system to assimilate the statistical regularities or consistencies in the domain and thus to generalize this information across items including novel forms (permitting transformation of nonwords, novel actions, etc.). The result is that, for items which correspond to the typical patterns within the domain, these modality-specific components are most efficient and the additional support from interaction with semantics, although present, is largely superfluous. In contrast, the inherently less efficient computation of atypical transformations benefits significantly from interaction with the semantic system. When this semantic contribution is weakened by damage in patients (e.g., Woollams et al., 2007) or by appropriately localized rTMS in neurologically intact participants (Holland \& Lambon Ralph, 2010) or is intrinsically weak as for low imageability/abstract words (e.g., Strain et al., 1995), then (a) accuracy for these items is diminished and (b) the resultant errors reflect a more typical pattern for the domain (a type of regularization error; Patterson et al., 2006; Patterson, Suzuki, Wydell, \& Sasanuma, 1995).

\section{Acknowledgments}

This work was supported by an MRC program grant to M. A. L. R. (G0501632 and MR/J004146/1); a Royal Society (UK) International Joint Grant awarded to M. A. L. R. and S. S.; and a Grantin-Aid for Scientific Research (22530794) from the Ministry of Education, Culture, Sports, Science, and Technology in Japan to S. S. T. U. was also supported by two fellowships: a scholarship from the Overseas Research Students Awards Scheme in the United Kingdom and an Overseas Study Fellowship from the Nakajima Foundation in Japan.

Reprint requests should be sent to Prof. Matthew A. Lambon Ralph, Neuroscience and Aphasia Research Unit, School of Psychological Sciences, Zochonis Building (T18), University of Manchester, Oxford Road, Manchester, M13 9PL, UK, or via e-mail: matt.lambon-ralph@manchester.ac.uk.

\section{REFERENCES}

Akinaga, K., \& Kindaichi, H. (2001). Shinmeikai Nibongo accent jiten [Shinmeikai Japanese accent dictionary]. Tokyo: Sanseido.

Amano, S., \& Kondo, T. (1999). Japanese NTT database series: Lexical properties of Japanese (1). Tokyo: Sanseido.

Amano, S., \& Kondo, T. (2000). Japanese NTT database series: Lexical properties of Japanese, word-frequency (11). Tokyo: Sanseido.

Behrmann, M., Nelson, J., \& Sekuler, E. (1998). Visual complexity in letter-by-letter reading: "Pure" alexia is not so pure. Neuropsychologia, 36, 1115-1132.

Botvinick, M. M., \& Plaut, D. C. (2006). Short-term memory for serial order: A recurrent neural network model. Psychological Review, 113, 201-233.
Butler, R., Patterson, K., \& Woollams, A. M. (2012). In search of meaning: Semantic effects on past-tense inflection. Quarterly Journal of Experimental Psychology, 65, 1633-1656.

Crisp, J., \& Lambon Ralph, M. A. (2006). Unlocking the nature of the phonological-deep dyslexia continuum: The keys to reading aloud are in phonology and semantics. Journal of Cognitive Neuroscience, 18, 348-362.

Cutler, A., \& Otake, T. (1994). Mora of phoneme? Further evidence for language-specific listening. Journal of Memory and Language, 33, 824-844.

Dell, G. S., Schwartz, M. F., Martin, N., Saffran, E. M., \& Gagnon, D. A. (1997). Lexical access in aphasic and nonaphasic speakers. Psychological Review, 104, 801-838.

Dilkina, K., McClelland, J. L., \& Plaut, D. C. (2008). A single-system account of semantic and lexical deficits in five semantic dementia patients. Cognitive Neuropsychology, 25, 136-164.

Evans, G., Lambon Ralph, M., \& Woollams, A. (2012). What's in a word? A parametric study of semantic influences on visual word recognition. Psychonomic Bulletin \& Review, 19, 325-331.

Fridriksson, J., Kjartansson, O., Morgan, P. S., Hjaltason, H., Magnusdottir, S., Bonilha, L., et al. (2010). Impaired speech repetition and left parietal lobe damage. Journal of Neuroscience, 30, 11057-11061.

Fushimi, T., Komori, K., Ikeda, M., Lambon Ralph, M. A., \& Patterson, K. (2009). The association between semantic dementia and surface dyslexia in Japanese. Neuropsychologia, 47, 1061-1068.

Graham, N. L., Patterson, K., \& Hodges, J. R. (2000). The impact of semantic memory impairment on spelling: Evidence from semantic dementia. Neuropsychologia, 38, 143-163.

Harm, M. W., \& Seidenberg, M. S. (2004). Computing the meanings of words in reading: Cooperative division of labor between visual and phonological processes. Psychological Review, 111, 662-720.

Hickok, G., \& Poeppel, D. (2007). The cortical organization of speech processing. Nature Reviews Neuroscience, 8, 393-402.

Holland, R., \& Lambon Ralph, M. A. (2010). The anterior temporal lobe semantic hub is a part of the language neural network: Selective disruption of irregular past tense verbs by rTMS. Cerebral Cortex, 20, 2771-2775.

Hulme, C., Roodenrys, S., Schweickert, R., Brown, G. D. A., Martin, S., \& Stuart, G. (1997). Word-frequency effects on short-term memory tasks: Evidence for a redintegration process in immediate serial recall. Journal of Experimental Psychology: Learning Memory and Cognition, 23, 1217-1232

Ijuin, M., Fushimi, T., Patterson, K., \& Tatsumi, I. (1999). A connectionist approach to Japanese kanji word naming. Psychologia, 42, 267-280.

Jefferies, E., Crisp, J., \& Lambon Ralph, M. A. (2006). The impact of phonological or semantic impairment on delayed auditory repetition: Evidence from stroke aphasia and semantic dementia. Aphasiology, 20, 963-992.

Jefferies, E., Frankish, C., \& Lambon Ralph, M. A. (2006). Lexical and semantic influences on item and order memory in immediate serial recognition: Evidence from a novel task. Quarterly Journal of Experimental Psychology, 59, 949-964.

Joanisse, M. F., \& Seidenberg, M. S. (1999). Impairments in verb morphology after brain injury: A connectionist model. Proceedings of the National Academy of Sciences, U.S.A. 96, 7592-7597. 
Kawamoto, A. H., Kello, C. T., Jones, R., \& Bame, K. (1998). Initial phoneme versus whole-word criterion to initiate pronunciation: Evidence based on response latency and initial phoneme duration. Journal of Experimental Psychology: Learning, Memory, and Cognition, 24, 862-885.

Knott, R., Patterson, K., \& Hodges, J. R. (1997). Lexical and semantic binding effects in short-term memory: Evidence from semantic memory. Cognitive Neuropsychology, 8, $1165-1216$.

Kubozono, H. (2006). The law of accent [written in Japanese]. Tokyo: Iwanami Shoten.

Lambon Ralph, M. A., Sage, K., Green Heredia, C., Berthier, M. L., Martínez-Cuitiño, M., Torralva, T., et al. (2011). El-La: The impact of degraded semantic representations on knowledge of grammatical gender in semantic dementia. Acta Neuropsychologica, 9, 115-132.

Masuda-Katsuse, I. (2006). Contribution of pitch-accent information to Japanese spoken-word recognition. Acoustical Science and Technology, 27, 97-103.

McClelland, J. L., St John, M., \& Taraban, R. (1989). Sentence comprehension: A parallel distributed processing approach. Language and Cognitive Processes, 4, SI287-SI336.

Minematsu, N., \& Hirose, K. (1995). Role of prosodic features in the human process of perceiving words and sentences in Japanese. Journal of the Acoustical Society of Japan, 16, 311-320.

Mirković, J., Seidenberg, M. S., \& Joanisse, M. F. (2011). Rules versus statistics: Insights from a highly inflected language. Cognitive Science, 35, 638-681.

Nozari, N., Kittredge, A. K., Dell, G. S., \& Schwartz, M. F. (2010). Naming and repetition in aphasia: Steps, routes, and frequency effects. Journal of Memory and Language, 63, 541-559.

O'Reilly, R. C. (2006). Biologically based computational models of high-level cognition. Science, 314, 91-94.

Otake, T., Hatano, G., Cutler, A., \& Mehler, J. (1993). Mora or syllable? Speech segmentation in Japanese. Journal of Memory and Language, 32, 258-278.

Paivio, A., \& Madigan, S. A. (1968). Imagery and association value in paired-associate learning. Journal of Experimental Psychology, 76, 35-39.

Parker, G. J. M., Luzzi, S., Alexander, D. C., Wheeler-Kingshott, C. A. M., Clecarelli, O., \& Lambon Ralph, M. A. (2005). Lateralization of ventral and dorsal auditory-language pathways in the human brain. Neuroimage, 24, 656-666.

Patterson, K., \& Lambon Ralph, M. A. (1999). Selective disorders of reading? Current Opinion in Neurobiology, 9, 235-239.

Patterson, K., Lambon Ralph, M. A., Hodges, J. R., \& McClelland, J. L. (2001). Deficits in irregular past-tense verb morphology associated with degraded semantic knowledge. Neuropsychologia, 39, 709-724.

Patterson, K., Lambon Ralph, M. A., Jefferies, E., Woollams, A. M., Jones, R., Hodges, J. R., et al. (2006). "Presemantic" cognition in semantic dementia: Six deficits in search of an explanation. Journal of Cognitive Neuroscience, 18, 169-183.

Patterson, K., \& Marcel, A. (1992). Phonological ALEXIA or PHONOLOGICAL alexia? In J. Alegria, D. Holender, J. Juncas de Morais, \& M. Radeau (Eds.), Analytic approaches to buman cognition (pp. 257-274). Amsterdam: North-Holland.

Patterson, K., Suzuki, T., Wydell, T., \& Sasanuma, S. (1995). Progressive aphasia and surface alexia in Japanese. Neurocase, 1, 155-165.

Pinker, S. (1991). Rules of language. Science, 253, 530-535.

Plaut, D. C., \& Kello, C. T. (1999). The emergence of phonology from the interplay of speech comprehension and production: A distributed connectionist approach. In B. MacWhinney
(Ed.), Emergence of language (pp. 381-415). Mahwah, NJ: Erlbaum.

Plaut, D. C., McClelland, J. L., Seidenberg, M. S., \& Patterson, K. (1996). Understanding normal and impaired word reading: Computational principles in quasi-regular domains. Psychological Review, 103, 56-115.

Rapcsak, S. Z., Beeson, P. M., Henry, M. L., Leyden, A., Kim, E., Rising, K., et al. (2009). Phonological dyslexia and dysgraphia: Cognitive mechanisms and neural substrates. Cortex, 45 , 575-591.

Rauschecker, J. P., \& Scott, S. K. (2009). Maps and streams in the auditory cortex: Nonhuman primates illuminate human speech processing. Nature Neuroscience, 12, $718-724$

Rogers, T. T., Lambon Ralph, M. A., Garrard, P., Bozeat, S., McClelland, J. L., Hodges, J. R., et al. (2004). Structure and deterioration of semantic memory: A neuropsychological and computational investigation. Psychological Review, 111, 205-235.

Rogers, T. T., Lambon Ralph, M. A., Hodges, J. R., \& Patterson, K. (2004). Natural selection: The impact of semantic impairment on lexical and object decision. Cognitive Neuropsychology, 21, 331-352.

Rohde, D. L. T. (1999). LENS: The light, efficient network simulator. Technical Report CMU-CS-99-164. Pittsburgh, PA: Carnegie Mellon University, Department of Computer Science.

Sakono, S., Ito, T., Fukuda, S. E., \& Fukuda, S. (2011). The effect of word accent production on reading performance in Japanese young children. Asia Pacific Journal of Speech, Language, and Hearing, 14, 51-60.

Sato, H. (1993). Accent analysis of common Japanese words [written in Japanese]. The Journal of the Acoustical Society of Japan, 49, 775-784.

Saur, D., Kreher, B. W., Schnell, S., Kümmerer, D., Kellmeyer, P., Vry, M. S., et al. (2008). Ventral and dorsal pathways for language. Proceedings of the National Academy of Sciences, U.S.A., 105, 18035-18040.

Seidenberg, M. S. (1997). Language acquisition and use: Learning and applying probabilistic constraints. Science, 275, 1599-1603.

Seidenberg, M. S., \& MacDonald, M. C. (1999). A probabilistic constraints approach to language acquisition and processing. Cognitive Science, 23, 569-588.

Seidenberg, M. S., \& McClelland, J. L. (1989). A distributed, developmental model of word recognition and naming. Psychological Review, 96, 523-568.

Shibahara, N., Zorzi, M., Hill, M. P., Wydell, T. N., \& Butterworth, B. (2003). Semantic effects in word naming: Evidence from English and Japanese kanji. Quarterly Journal of Experimental Psychology: Section A-Human Experimental Psychology, 56, 263-286.

Strain, E., Patterson, K., \& Seidenberg, M. S. (1995). Semantic effects in single-word naming. Journal of Experimental Psychology: Learning Memory and Cognition, 21, 1140-1154.

Tamaoka, K., \& Makioka, S. (2004). Frequency of occurrence for units of phonemes, morae, and syllables appearing in a lexical corpus of a Japanese newspaper. Behavior Research Methods Instruments \& Computers, 36, 531-547.

Tyler, L. K., Voice, J. K., \& Moss, H. E. (2000). The interaction of meaning and sound in spoken word recognition. Psychonomic Bulletin \& Review, 7, 320-326.

Ueno, T., Saito, S., Rogers, T. T., \& Lambon Ralph, M. A. (2011). Lichtheim 2: Synthesizing aphasia and the neural basis of language in a neurocomputational model of the dual dorsal-ventral language pathways. Neuron, 72, 385-396. 
Welbourne, S. R., Woollams, A. M., Crisp, J., \& Lambon Ralph, M. A. (2011). The role of plasticity-related functional reorganization in the explanation of central dyslexias. Cognitive Neuropsychology, 28, 65-108.

Woollams, A. M., Joanisse, M., \& Patterson, K. (2009). Past-tense generation from form versus meaning: Behavioural data and simulation evidence. Journal of Memory and Language, 61, 55-76.

Woollams, A. M., Lambon Ralph, M. A., Plaut, D. C., \& Patterson, K. (2007). SD-squared: On the association between semantic dementia and surface dyslexia. Psychological Review, 114, 316-339.

Yang, J., McCandliss, B. D., Shu, H., \& Zevin, J. D. (2009). Simulating language-specific and languagegeneral effects in a statistical learning model of Chinese reading. Journal of Memory and Language, 61, 238-257.

Yuzawa, M. (2002). Effects of pitch accent on short-term retention of words in young children [written in Japanese]. Shinrigaku Kenkyu, 73, 258-263. 